\title{
Diagnostic Accuracy of Peritoneal Fluid GeneXpert in the Diagnosis of Intestinal Tuberculosis, Keeping Histopathology as the Gold Standard
}

Raheel Ahmad ${ }^{1}$, Mehwish Changeez ${ }^{1}$, Jahangir S. Khan ${ }^{1}$, Usman Qureshi ${ }^{1}$, Maham Tariq ${ }^{1}$, Sara Malik ${ }^{1}$, Sheikh Haseeb Ahmad ${ }^{1}$, Muhammad Salman Shafique ${ }^{1}$

1. Surgery, Holy Family Hospital, Rawalpindi, PAK

Corresponding author: Mehwish Changeez, mehwishchangez@gmail.com

\section{Abstract}

\section{Background}

The diagnosis of abdominal tuberculosis is a major health challenge. Limited data are available to support the use of GeneXpert MTB/RIF in the diagnosis of abdominal tuberculosis. The current study is an analysis of the sensitivity and specificity of GeneXpert MTB/RIF for the diagnosis of abdominal tuberculosis, keeping histopathology as the gold standard.

\section{Materials and methods}

A prospective study was conducted in Surgery Unit-I of Holy Family hospital in the year 2017. Data of 21 patients presenting with abdominal tuberculosis were collected. The samples collected were ascitic fluid for GeneXpert and acid-fast bacilli (AFB) and a tissue sample for histopathology, which included either the enlarged lymph nodes or the involved gut segment.

\section{Results}

Out of a total of 21 patients, 10 were male and 11 were female. The predominant age group was less than 30 years with $76.2 \%$ cases. Of the 21 samples analyzed, all were positive for tuberculosis (TB) by histopathology. GeneXpert was positive in six and negative in 15 patients. The sensitivity of GeneXpert was $28.57 \%$ and specificity was $0 \%$. The positive predictive value was $100 \%$. The diagnostic accuracy was found to be $28.57 \%$.

\section{Conclusion}

Received 09/25/2018

Review began 10/05/2018

Review ended 10/13/2018

Published 10/15/2018

\section{() Copyright 2018}

Ahmad et al. This is an open access article distributed under the terms of the Creative Commons Attribution License CC-BY 3.0., which permits unrestricted use, distribution, and reproduction in any medium, provided the original author and source are credited.
In our study, GeneXpert has shown poor sensitivity and specificity for the detection of abdominal TB from ascitic fluid samples. On the basis of this data, we lay stress on finding new tests and biomarkers for the rapid diagnosis of abdominal $\mathrm{TB}$.

Categories: Infectious Disease, Healthcare Technology

Keywords: abdominal tuberculosis, genexpert

\section{Introduction}

Tuberculosis is a significant health problem all over the world, especially in developing countries [1-2]. In 2013, nine million people became infected with tuberculosis and 1.5 million people died because of this disease all over the world [3]. Pakistan is ranked fifth among the high burden countries with tuberculosis with an annual 510,000 new cases [4]. A lack of typical clinical features led to a delay in diagnosis and the management of extrapulmonary tuberculosis [5].

Once the diagnosis has been considered, it is confirmed by using various tests. Culture is considered the gold standard for the diagnosis of Mycobacterium tuberculosis (MTB) but takes up to two to eight weeks. Microscopy for the detection of acid fast bacilli (AFB) is an inexpensive and rapid test but has low sensitivity and specificity and is also unable to differentiate between tuberculous and non-tuberculous mycobacterium (NTM). A histological examination of tissue is unable to differentiate tuberculosis from other diseases like sarcoidosis and NTM, however, they can detect stained tubercle bacilli. Other tests employed for the diagnosis of extrapulmonary tuberculosis (EPTB) include serological assays, Mantoux test, and polymerase chain reaction (PCR) assays. These tests vary in their sensitivity and specificity and some of these tests require relatively longer time [6-7].

The GeneXpert MTB/RIF system offers a rapid and efficient technique, which can detect Mycobacterium 


\section{Cureus}

tuberculosis and drug resistance to rifampicin simultaneously within two hours [2]. The system is easy to use, carries the minimum risk of cross-contamination and is biosafe. World Health Organization (WHO) approved GeneXpert MTB/RIF in 2011 and recommended it for prompt implementation [8]. A recently published systemic review showed a pooled sensitivity of $88 \%$ and a pooled specificity of $98 \%$ [9] for the diagnosis of pulmonary tuberculosis. Another study assessed the convenience of an Xpert assay in EPTB and showed an $81.3 \%$ sensitivity and $99.8 \%$ specificity, considering culture and clinical diagnosis as the gold standard [10], however, still there is limited evidence to use GeneXpert in the diagnosis of EPTB [11].

The purpose of this study is to evaluate the sensitivity and specificity of GeneXpert MTB/RIF in the diagnosis of abdominal tuberculosis in comparison with histopathology as the standard with the aim of establishing the most appropriate laboratory tests algorithm on the basis of available knowledge and techniques.

\section{Materials And Methods}

A prospective study was conducted in Surgery Unit-I of Holy Family Hospital, where the data of 21 patients presenting with abdominal tuberculosis in the year 2017 was collected. A detailed history of clinical symptoms, a past history of tuberculosis, or the use of anti-tuberculous drugs and TB contact was taken. All patients presenting with a suspected complication of intestinal tuberculosis were investigated and managed by surgical intervention after initial resuscitation. Samples were collected from all patients undergoing laparotomy and operative findings were noted. The samples collected were ascitic fluid for GeneXpert and AFB and tissue samples for histopathology, which included either the enlarged lymph nodes or the involved gut segment.

Data were recorded and analyzed using Statistical Package for Social Sciences (SPSS) v20.0 (SPSS Inc., Chicago, IL, USA). For categorical variables, frequencies and percentages were reported. Sensitivity and specificity were calculated for GeneXpert, keeping histopathology as the standard.

True positive (TP): This was labeled if peritoneal fluid GeneXpert was positive and histopathology was also positive.

False positive (FP): This was labeled if peritoneal fluid GeneXpert was positive but histopathology was negative.

True negative (TN): This was labeled if peritoneal fluid GeneXpert was negative and histopathology was also negative.

False negative (FN): This was labeled if peritoneal fluid GeneXpert was negative but histopathology was positive.

\section{Results}

A total of 21 patients were included in our study out of which 10 were male and 11 were female. The predominant age group was less than 30 years with $76.2 \%$ cases (Table 1 ). The most common presenting symptoms were abdominal pain, constipation, and vomiting in $38.1 \%$ patients. Constitutional symptoms of weight loss and fever were seen in $47.6 \%$ patients. A history of pulmonary tuberculosis and the use of antitubercular treatment (ATT) was positive in $57.1 \%$ and $47.6 \%$ patients, respectively.

\begin{tabular}{|c|c|c|}
\hline Age & Frequency $(n=21)$ & Percentage \\
\hline Less than 30 years & 16 & 16.2 \\
\hline more than 30 years & 5 & 28.6 \\
\hline
\end{tabular}

\section{TABLE 1: Age groups}

The most common preoperative finding was intestinal perforation with intestinal mass formation (33.3\%) followed by intestinal perforation and stricture formation as shown in Table 2 . 


\section{Cureus}

\begin{tabular}{|l|l|l|}
\hline Per op findings & Frequency $(\mathbf{n}=21)$ & Percentage \\
\hline Intestinal perforation & 5 & 23.8 \\
\hline Mass & 4 & 19 \\
\hline Stricture & 5 & 23.8 \\
\hline Intestinal perforation with mass formation & 7 & 33.3 \\
\hline
\end{tabular}

\section{TABLE 2: Per op findings}

Resection and stoma formation was the most commonly performed procedure(42.9\%) as seen in Table 3.

\begin{tabular}{|l|l|l|}
\hline Surgical procedure & Frequency $(\mathbf{n}=21)$ & Percentage \\
\hline Resection and stoma formation & 9 & 42.9 \\
\hline Stoma formation & 6 & 28.6 \\
Stricturoplasty & 3 & 14.3 \\
\hline Biopsy only & 3 & 14.3 \\
\hline
\end{tabular}

TABLE 3: Surgical procedures performed

Of the 21 samples analyzed, all were positive for TB by histopathology. GeneXpert gave six positive and 15 negative results. However, for six cases, both histopathology and GeneXpert were positive. The sensitivity of GeneXpert was $28.57 \%$ and specificity was $0 \%$. The positive predictive value was $100 \%$. Diagnostic accuracy was found to be $28.57 \%$ (Tables $4-5$ ).

\begin{tabular}{|l|l|l|}
\hline GeneXpert & Frequency $(\mathbf{n}=\mathbf{2 1})$ & Percentage \\
\hline Positive & 6 & 28.57 \\
Negative & 15 & 71.43 \\
\hline
\end{tabular}

TABLE 4: Results of GeneXpert

\begin{tabular}{|c|c|c|c|}
\hline PERITONEAL FLUID GENEXPERT & Histopathology POSITIVE & Histopathology NEGATIVE & Total \\
\hline Positive & 6 (True positive) & 0 (False positive) & 6 \\
\hline Negative & 15 (False negative) & 0 (True negative) & 15 \\
\hline Total & 21 & 0 & 21 \\
\hline
\end{tabular}

\section{TABLE 5: Contingency table for GeneXpert}

\section{Discussion}

Pulmonary tuberculosis is the most common form of tuberculosis seen worldwide. Extrapulmonary tuberculosis involving lymph nodes, meninges, the intestine, bones, joints, genitourinary tract, etc. is also associated with significant morbidity and mortality. The abdomen is the sixth-most common site of extrapulmonary tuberculosis. The incidence of abdominal tuberculosis is increasing all over the world. However, very little knowledge and literature have been updated and it is still a diagnostic dilemma [12]. 
In 2013, WHO introduced the use of the GeneXpert MTB/RIF assay on pulmonary samples, where it has high sensitivity and specificity, and has thus been recommended for national TB programs in developing countries [1]. However, information regarding the performance of the MTB/RIF assay on extrapulmonary samples is still emerging. It has been granted as a conditional recommendation for the diagnosis of EPTB, however, the overall evidence has been cited as insufficient [13].

Previous studies of the MTB/RIF assay have reported a sensitivity of $100 \%$ for smear-positive respiratory and non-respiratory samples. Sensitivity for smear-negative samples was 57\% and 37\%, respectively [14]. Another study conducted by Zeka et al. showed an improved sensitivity of $100 \%$ for smear-positive extrapulmonary tuberculosis and 63\% for smear-negative EPTB [15].

In our study, the sensitivity of GeneXpert for the diagnosis of abdominal tuberculosis was $28.57 \%$. The positive predictive value and diagnostic accuracy were found be $100 \%$ and $28.57 \%$, respectively. Similar low sensitivity on ascitic fluid samples (27.8\%) was reported by the study conducted by Alvereza et al [16].

A study conducted by SB Rufai assessed the sensitivity of the MTB/RIF assay for the diagnosis of tuberculosis by using ascetic fluid samples, keeping MGIT-960 as the gold standard. Out of 67 patients, the MTB/RIF assay was positive in only 12 (17.9\%) cases while $82.1 \%$ was negative. The study showed that the diagnostic yield of the MTN/RIF assay was low even in culture-positive specimens (70.5\%), indicating that in highly proteinous body fluids, such as ascitic fluid, Xpert MTB/RIF-negative cases must be investigated further using other phenotypic methods [1]. Xpert MTB/RIF has, however, a high positive predictive value (PPV), meaning that if GeneXpert is positive, the ATT can be started without waiting for other investigations.

A study conducted by Grant Theron on the determinants of PCR performance concluded that a low mycobacillary load in extrapulmonary samples as compared to pulmonary specimens is primarily responsible for the low sensitivity of the MTB/RIF assay in the diagnosis of extrapulmonary tuberculosis [13].

The low sensitivity of GeneXpert for the diagnosis of abdominal tuberculosis in our study can be attributed to the use of ascitic fluid as a specimen, small sample size, and poor sample-handling techniques.

\section{Conclusions}

Even though the MTB/RIF assay has high sensitivity for the detection of pulmonary tuberculosis, in our study, GeneXpert evaluated for the detection of abdominal TB from ascitic fluid samples has shown poor sensitivity. On the basis of this data, therefore, we lay stress on finding new tools and discovering new biomarkers for the rapid diagnosis of abdominal TB.

\section{Additional Information \\ Disclosures}

Human subjects: Consent was obtained by all participants in this study. Research and Ethical Committee Rawalpindi Medical University and Allied Hospitals, Rawalpindi issued approval RMU/16-148. The research titled " Diagnostic Accuracy of Peritoneal Fluid GeneXpert in the Diagnosis of Intestinal Tuberculosis Keeping Histopathology as the Gold Standard" conforms to the accepted ethical standards established by the Institution Research Forum of RMU. Therefore, the research proposal has been approved by the Research and Ethical Committee of RMU. Animal subjects: All authors have confirmed that this study did not involve animal subjects or tissue. Conflicts of interest: In compliance with the ICMJE uniform disclosure form, all authors declare the following: Payment/services info: All authors have declared that no financial support was received from any organization for the submitted work. Financial relationships: All authors have declared that they have no financial relationships at present or within the previous three years with any organizations that might have an interest in the submitted work. Other relationships: All authors have declared that there are no other relationships or activities that could appear to have influenced the submitted work.

\section{References}

1. Rufai SB, Singh S, Singh A, Kumar P, Singh J, Vishal A: Performance of Xpert MTB/RIF on ascitic fluid samples for detection of abdominal tuberculosis. J Lab Physicians. 2017, 9:47-52. 10.4103/09742727.187927

2. Vadwai V, Boehme C, Nabeta P, Shetty A, Alland D, Rodrigues C: Xpert MTB/RIF: a new pillar in diagnosis of extrapulmonary tuberculosis?. J Clin Microbiol. 2011, 49:2540-2545. 10.1128/JCM.02319-10

3. Moussa HS, Bayoumi FS, Mohamed AM: Gene Xpert for direct detection of Mycobacterium tuberculosis in stool specimens from children with presumptive pulmonary tuberculosis. Ann Clin Lab Sci. 2016, 46:198203.

4. Stop tuberculosis. (2018). Accessed: July 20, 2018: http://www.emro.who.int/tuberculosis/epidemiologicalsituation/epidemiological-situation.html.

5. Kumar S, Bopanna S, Kedia S, et al.: Evaluation of Xpert MTB/RIF assay performance in the diagnosis of abdominal tuberculosis. Intest Res. 2017, 15:187-194. Accessed: July 20, 2018: http://irjournal.org/journal/view.php?doi=10.5217/ir.2017.15.2.187. 10.5217/ir.2017.15.2.187 
6. Maynard-Smith L, Larke N, Peters JA, Lawn SD: Diagnostic accuracy of the Xpert MTB/RIF assay for extrapulmonary and pulmonary tuberculosis when testing non-respiratory samples: a systematic review. BMC Infect Dis. 2014, 14:709. Accessed: July 21, 2018:

https://bmcinfectdis.biomedcentral.com/articles/10.1186/s12879-014-0709-7. 10.1186/s12879-014-0709-7

7. Pravin KN, Chourasia E: Use of GeneXpert assay for diagnosis of tuberculosis from body fluid specimens, a 2 years study. J Microbiol Biotechnol. 2016, 1:105. 10.23880/OAJMB-16000105

8. Singh UB, Pandey P, Mehta G, et al.: Genotypic, phenotypic and clinical validation of GeneXpert in extrapulmonary and pulmonary tuberculosis in India. PLoS One. 2016, $11: \mathrm{e} 0149258$. Accessed: July 20, 2018: https://www.ncbi.nlm.nih.gov/pubmed/26894283. 10.1371/journal.pone.0149258

9. Steingart KR, Sohn H, Schiller I, Kloda LA, Boehme CC, Pai M, Dendukuri N: Xpert ${ }^{\circledR}$ MTB/RIF assay for pulmonary tuberculosis and rifampicin resistance in adults. Cochrane Database Syst Rev. 2013, 1:9593. Accessed: July 20, 2018: https://www.ncbi.nlm.nih.gov/pubmed/24448973.

10.1002/14651858.CD009593.pub3

10. Tortoli E, Russo C, Piersimoni C, et al.: Clinical validation of Xpert MTB/RIF for the diagnosis of extrapulmonary tuberculosis. Eur Respir J. 2012, 40:442-447. 10.1183/09031936.00176311

11. Lawn SD, Zumla AI: Diagnosis of extrapulmonary tuberculosis using the Xpert ${ }^{\circledR}$ MTB/RIF assay . Expert Rev Anti Infect Ther. 2012, 10:631-635. 10.1586/eri.12.43

12. Jagielski T, Augustynowicz-Kopec E, Zwolska Z: Epidemiology of tuberculosis: a global, European and Polish perspective [Article in Polish]. Wiad Lek. 2010, 63:230-246.

13. Theron G, Peter J, Calligaro G, et al.: Determinants of PCR performance (Xpert MTB/RIF), including bacterial load and inhibition, for TB diagnosis using specimens from different body compartments. Sci Rep. 2014, 4:5658. Accessed: July 20, 2018: https://www.ncbi.nlm.nih.gov/pubmed/25014250. 10.1038/srep05658

14. Armand S, Vanhuls P, Delcroix G, Courcol R, Lemaitre N: Comparison of the Xpert MTB/RIF test with an IS6110-TaqMan real-time PCR assay for direct detection of Mycobacterium tuberculosis in respiratory and nonrespiratory specimens. J Clin Microbiol. 2011, 49:1772-1776. 10.1128/JCM.02157-10

15. Zeka AN, Tasbakan S, Cavusoglu C: Evaluation of the GeneXpert MTB/RIF assay for rapid diagnosis of tuberculosis and detection of rifampin resistance in pulmonary and extrapulmonary specimens. J Clin Microbiol. 2011, 49:4138-4141. 10.1128/JCM.05434-11

16. Alvarez-Uria G, Azcona JM, Midde M, Naik PK, Reddy S, Reddy R: Rapid diagnosis of pulmonary and extrapulmonary tuberculosis in HIV-infected patients. Comparison of LED fluorescent microscopy and the GeneXpert MTB/RIF assay in a district hospital in India. Tuberc Res Treat. 2012, 2012:932862. Accessed: July 21, 2018: https://www.hindawi.com/journals/trt/2012/932862/cta/. 10.1155/2012/932862 